Revue internationale P.M.E.

Économie et gestion de la petite et moyenne entreprise

Revue

internationale

PME

\title{
Le cyberentrepreneuriat : une étude exploratoire
}

\section{Camille Carrier, Louis Raymond et Anissa Eltaief}

Volume 15, numéro 3-4, 2002

URI : https://id.erudit.org/iderudit/1008816ar

DOI : https://doi.org/10.7202/1008816ar

Aller au sommaire du numéro

Éditeur(s)

Presses de l’Université du Québec

ISSN

0776-5436 (imprimé)

1918-9699 (numérique)

Découvrir la revue

Citer cette note

Carrier, C., Raymond, L. \& Eltaief, A. (2002). Le cyberentrepreneuriat : une étude exploratoire. Revue internationale P.M.E., 15(3-4), 139-162.

https://doi.org/10.7202/1008816ar

\section{Résumé de l'article}

Les nouvelles technologies de l'information sont à l'origine d'une nouvelle forme d'entrepreneuriat, en l'occurrence le cyberentrepreneuriat, basée sur les nouveaux modèles d'affaires électroniques. Comme il s'agit d'un phénomène encore en phase d'émergence, il n'est pas étonnant de constater qu'il n'existe encore que très peu d'études sur le sujet ; la présente étude vise à combler cette lacune. Cette étude exploratoire en profondeur de cinq cas de cyberentreprises permet de jeter un éclairage sur le processus de création de la cyberentreprise ainsi que sur les stratégies et facteurs de compétitivité privilégiés par les cyberentrepreneurs concernés. 


\title{
Note de recherche Le cyberentrepreneuriat: une étude exploratoire
}

\author{
Camille CARRIER \\ Louis RAYMOND
}

Université du Québec à Trois-Rivières

Anissa ELTAIEF

Université de Toulouse I

\section{MOTS CLÉS}

\section{Cyberentrepreneuriat - Entrepreneuriat - Cyberentrepreneurship Entrepreneurship - Commerce électronique - Internet}

\begin{abstract}
RÉSUMÉ
Les nouvelles technologies de l'information sont à l'origine d'une nouvelle forme d'entrepreneuriat, en l'occurrence le cyberentrepreneuriat, basée sur les nouveaux modèles d'affaires électroniques. Comme il s'agit d'un phénomène encore en phase d'émergence, il n'est pas étonnant de constater qu'il n'existe encore que très peu
\end{abstract}

LES AUTEURS

CAMILLE CARRIER, D.Sc., est professeure titulaire de management au Département des sciences de la gestion de l'Université du Québec à Trois-Rivières. Sa recherche a été publiée dans des revues telles que Entrepreneurship Theory and Practice, Journal of Small Business Management et International Small Business Journal.

LouIS RAYMOND, Ph.D., est titulaire de la Chaire de recherche du Canada sur la performance des entreprises et professeur titulaire de systèmes d'information au Département des sciences de la gestion de l'Université du Québec à Trois-Rivières. Sa recherche a été publiée dans des revues telles que MIS Quarterly, Journal of Management Information Systems et International Journal of Technology Management.

ANISSA ELTAIEF, M. Sc., est détentrice d'une maîtrise en gestion des PME de l'Université du Québec à Trois-Rivières et étudiante au doctorat en gestion à l'Université de Toulouse I. 
d'études sur le sujet; la présente étude vise à combler cette lacune. Cette étude exploratoire en profondeur de cinq cas de cyberentreprises permet de jeter un éclairage sur le processus de création de la cyberentreprise ainsi que sur les stratégies et facteurs de compétitivité privilégiés par les cyberentrepreneurs concernés.

\begin{abstract}
New information technologies have given rise to a new form of entrepreneurship, namely cyberentrepreneurship, based on the new electronic business models. Being an emerging phenomenon, there have been as of yet few studies on the subject. The present study aims to remedy this situation. An exploratory study of five cases sheds some light on the cyberenterprise creation process, and on the strategies and competitive factors focused upon by cyberentrepreneurs.
\end{abstract}

\title{
RESUMEN
}

Las nuevas technologias de información conducen a un nuevo tipo de empresas, las cyberempresas fundadas sobre los nuevos modelos de asuntos electrónicos. Dado que es un fenómeno emergente, hay pocos investigaciones sobre este asunto. La investigación presente intenta a corregir esta situación. Una exploración de cinco casos ilustra el proceso de creación de la cyberempresa, y las strategías y fuerzas concurrenciales privilegiadas por los creadores de ese tipo de empresas.

\section{ZUSAMMENFASSUNG}

Die neuen Informationstechnologien sind momentan der Ausgangspunkt für eine neue Form des Unternehmertums, im vorliegenden Fall das Cyber-Unternehmertum. Bis jetzt hat man sich stark für das Studium des Unternehmertums bei traditionellen Geschäftsmodellen interessiert. Das Cyber-Unternehmertum ist gewissermassen ein neu auftauchendes Phänomen und es ist nicht überraschend festzustellen, dass noch sehr wenige Studien zu diesem Forschungsobjekt existieren. Die vorliegende Studie hat zum Ziel, diese Lücke zu schliessen. Diese vertiefte Studie über fünf Fälle von Cyber-Unternehmen hat erlaubt, den Prozess des Wachstums und der Gründung der Cyber-Unternehmung aufzulichten sowohl die Strategien und die Faktoren der bevorzugten Wettbewerbsfähigkeit der betroffenen Cyber-Unternehmer aufzuzeigen.

\section{Introduction}

Comme le souligne Scott-Morton (1995), les technologies de l'information (TI) comptent parmi les éléments clés du bien-être économique mondial, car leur diffusion affecte profondément la majorité des industries et des services. Une nouvelle économie dans laquelle le savoir constitue la ressource stratégique la plus importante oblige de plus en plus d'entreprises à revoir leurs pratiques traditionnelles et à tirer profit d'outils informationnels puissants leur permettant d'effectuer 
des transactions commerciales à une échelle beaucoup plus vaste, de nouveaux partenariats et réseautages avec leurs clients et leurs fournisseurs, de mettre en place des systèmes de veille facilitant la détection de nouvelles occasions d'affaires et d'innovations (Raymond, 2000). Les technologies informationnelles « permettent à l'entreprise de transcender les barrières spatiales et temporelles » (O'Brien, 1999).

Ces nouvelles technologies sont également en voie de donner naissance à de nouvelles formes entrepreneuriales, plus particulièrement au cyberentrepreneuriat. Le cyberentrepreneur crée une entreprise fondée essentiellement sur l'utilisation du commerce électronique, dont les activités principales reposent sur l'exploitation des réseaux basés sur les technologies Internet, les intranets et les extranets (Bret et Champeaux, 2000). Le commerce électronique comprend des fonctions de support à l'échange d'information et aux transactions commerciales apportant généralement des avantages aux entreprises. Il consistait, à l'origine, en un échange de données informatisé (EDI) sur des réseaux de communication à valeur ajoutée, généralement imposé par de grandes organisations et basé sur des spécifications rigides et relativement complexes. Mais le commerce électronique a rapidement évolué vers l'Internet et le World Wide Web, soit une infrastructure de communication et de transaction plus « ouverte » (Raymond, 2001).

Le cyberentrepreneuriat semble constituer une voie prometteuse pour plusieurs individus soucieux d'innover et d'exploiter les possibilités qu'offre le développement des nouvelles technologies de l'information. On pourrait même être tenté d'affirmer qu'il est fortement représentatif de l'entrepreneuriat dans sons sens le plus noble puisque Shane et Venkataraman (2000) placent la découverte et l'exploitation d'occasions d'affaires profitables au cœur même de ce phénomène. Depuis Schumpeter (1959) jusqu'à Kirzner (1979), l'innovation et l'opportunité d'affaires ont également été décrits comme constituant l'essence même de l'entrepreneuriat. Or, certains auteurs présentent le cyberentrepreneuriat comme une pratique d'affaires innovatrice pouvant permetttre de mieux détecter et de mieux saisir les occasions d'affaires.

On s'est beaucoup intéressé jusqu'à maintenant à l'étude de l'entrepreneuriat qui s'exerce dans des modèles d'affaires plus traditionnels ${ }^{1}$. Précisons d'emblée que l'entrepreneuriat est ici associé à la création de nouvelles entreprises et non au développement d'activités nouvelles dans les organisations existantes. Comme le précise Verstraete (2001), «il n’y a pas de consensus sur une théorie de l'entrepreneuriat, encore moins une définition univoque ». Le cyberentrepreneuriat s'avérant un phénomène encore en émergence, il n'est pas étonnant de constater

1. À cet égard, consulter le numéro spécial de la revue Entrepreneurship Theory and Practice (2001), vol. 25, $\mathrm{n}^{\circ}$ 4, qui fait le bilan de la recherche sur l'entrepreneuriat; voir également l'ouvrage de Verstraete (2000).

Revue internationale P.M.E., vol. 15, nos 3-4, 2002 
qu'on connaît encore bien mal le cyberentrepreneur et les caractéristiques de ses processus de création d'affaires. Partant d'une perspective interprétativiste et d'une démarche holistico-inductive, nulle hypothèse d'une spécificité intrinsèque du cyberentrepreneuriat n'est ici faite. L'objectif est plutôt d'approfondir les connaissances sur l'entrepreneuriat à travers une nouvelle manifestation de ce phénomène. Qui est le cyberentrepreneur? Comment en arrive-t-il à identifier et à exploiter une idée d'affaires intéressante? Quelles sont ses stratégies et comment définit-il les bases de la compétitivité de son affaire ? Voilà autant de questions que la présente recherche veut explorer à travers l'étude de cinq cas de cyberentreprises.

\section{Une littérature encore peu abondante sur le sujet}

Comme nous l'avons déjà mentionné, les études réalisées sur l'entrepreneuriat portent presque en totalité sur les contextes d'affaires traditionnels. À part quelques écrits à caractère plus populaire visant surtout à présenter l'émergence du phénomène ou à rapporter les succès particulièrement remarquables, nous n'avons pas relevé d'études empiriques sur cette nouvelle forme d'entrepreneuriat qu'est le cyberentrepreneuriat.

Dans un tel contexte, il a tout de même été jugé approprié de consulter les écrits traitant de l'entrepreneuriat technologique, forme entrepreneuriale qui se rapproche probablement le plus du cyberentrepreneuriat dans la mesure où il s'agit essentiellement de créations d'entreprise fondées sur une nouvelle technologie. Selon Blais et Toulouse (1992), les entrepreneurs technologiques possèdent des caractéristiques particulières. Leur étude démontre que ce sont des individus dont la moyenne d'âge est d'environ 30 ans au moment de la création de leur entreprise, ce qui est très jeune. Ayant complété des études universitaires avancées, ils ont aussi acquis une excellente expérience de la technologie qu'ils utilisent; ces entrepreneurs maîtrisent donc très bien leur domaine. Sur le plan des traits personnels, ils semblent empreints d'une passion remarquable pour leur technologie tout en étant des innovateurs résolus.

Toujours selon Blais et Toulouse (1992), ces entrepreneurs technologiques se distinguent par l'acuité de leur perception des besoins du marché et par le pragmatisme des solutions qu'ils développent pour y répondre. Ces entrepreneurs sont également généralement des administrateurs patients qui acceptent très sereinement l'absence de profits immédiats, compte tenu des investissements financiers requis et des longues périodes de développement et d'ajustement du produit technologique. Ils ont une vision à très long terme et, étant donné leur capacité à différer l'anticipation de récompenses tangibles, visent d'abord et avant tout une croissance à long terme. 
Sur un plan plus managérial, on a établi que les entrepreneurs ont tendance à présenter un profil stratégique particulier. Plusieurs typologies ont été développées pour caractériser les entrepreneurs au plan stratégique. Ayant effectué une synthèse de ces typologies, Filion (2000) indique qu'une des plus classiques - et, qui plus est, vérifiée -, est celle de Smith (1967). Cette typologie relevait la présence d'une différence importante entre l'entrepreneur artisan et l'entrepreneur opportuniste ou d'affaires, ce dernier présentant une orientation vers la croissance beaucoup plus définie que le premier. Il semble toutefois que Smith, dès l'origine de cette typologie, ait jugé l'entrepreneur technologique comme faisant partie d'une catégorie à part. La plupart des typologies qui ont suivi par la suite ${ }^{2}$ vont plus ou moins dans le même sens. C'est le cas, par exemple, de celle de Julien et Marchesnay (1996) qui classifie les entrepreneurs à partir de trois grandes aspirations socioéconomiques de l'entrepreneur, en l'occurrence la pérennité, l'indépendance et la croissance. Partant de là, ces auteurs présentent deux grands profils stratégiques de l'entrepreneur. Le premier, le PIC (pérennité - indépendance croissance) se situe dans une logique patrimoniale et accorde énormément d'importance à l'accumulation d'actifs ayant valeur de cession ou d'usage, mettant par conséquent l'accent sur la pérennité de son entreprise; à l'inverse, le CAP (croissance - autonomie - pérennité) est animé par une logique entrepreneuriale qui le pousse à privilégier d'abord et avant tout les activités à croissance forte.

Il est plausible de croire que le cyberentrepreneur puisse avoir certains traits similaires à ceux de l'entrepreneur technologique, mais il importe en même temps de réaliser que leurs contextes d'opération sont généralement fort différents. En effet, le premier innove en utilisant la technologie pour faire connaître et distribuer son produit tandis que, pour le deuxième, la technologie n'est plus un outil de communication ou de diffusion mais constitue le produit ou le procédé en luimême. Il serait conséquemment difficile d'associer plus directement et étroitement ces deux entrepreneurs. En revanche, sur le plan de la logique d'action stratégique, on peut supposer que le cyberentrepreneur démontre d'emblée une assez forte orientation vers le risque, la recherche d'occasions d'affaires et la croissance.

Sur le plan des processus de création et de développement des entreprises à caractère technologique, on prendra également connaissance des travaux de Samson (1990) et de ceux de Blair et Scott (1979). Cependant, encore ici, il semble difficile de transposer les théories de l'approche entrepreneuriale utilisée par l'entrepreneur qui se concentre sur un produit technologique au contexte particulier du cyberentrepreneur pour qui la technologie n'est pas une fin mais plutôt un moyen.

2. Voir par exemple celles de Miles et Snow (1978), Lafuente et Salas (1989) et Filion (1996). 


\section{Présentation de l'étude}

Dans un contexte où l'on ne dispose encore d'aucune étude empirique sur le phénomène de la cyberentreprise, une recherche à caractère d'abord exploratoire est apparue plus appropriée. En effet, comme le précise Eisenhardt (1989), une stratégie inductive à partir de l'observation d'une réalité apparaît nécessaire lorsque l'objet d'étude visé est relativement nouveau. L'étude de cas a donc été retenue comme méthode de recherche puisqu'elle permettait, dans les circonstances, de documenter en profondeur les expériences des cyberentreprises étudiées. Selon Eisenhardt (1989) et Maxwell (1996), les cas doivent être choisis en fonction de leur degré de représentativité du phénomène étudié.

Étant donné que le cyberentrepreneuriat n'est pas rattaché à un domaine industriel en particulier, nous avons sélectionné cinq cas de cyberentreprises œuvrant dans des secteurs d'activité différents. De plus, des modèles d'affaires également différents ont été recherchés, c'est-à-dire des cas de commerce électronique « B2B » (entreprise à entreprise) et des cas de « B2C » (entreprise à consommateur). Ces cas ont été choisis parmi la population des cyberentreprises qui sont répertoriées dans des engins de recherche tel Yahoo. En ce qui concerne les critères de sélection, ils ont été établis à partir de la structure des pages Web des entreprises. Pour les cas de « B2C » ont été sélectionnées des entreprises présentant une vitrine électronique avec des modules de paiement électronique et qui offraient des produits sous une forme numérique, c'est-à-dire directement transmissibles par l'Internet tels que documents et logiciels. Pour les cas de «B2B » étaient privilégiées des entreprises offrant un service à toutes les étapes du cycle commercial par le biais d'Internet et dont c'était l'activité principale plutôt qu'un service secondaire ou annexe.

Conséquemment, des entrevues semi-structurées ont été menées auprès des propriétaires-dirigeants de cinq cyberentreprises (toutes des PME) de la région du grand Montréal. Le questionnaire uniforme ayant servi à l'entrevue avait pour but de mieux connaître ces entrepreneurs, de les amener à décrire le processus suivi pour créer leur entreprise et les bases de leur stratégie de compétitivité. Chaque entrevue a duré environ deux heures et chacune a été enregistrée sur magnétophone. Par la suite, les matériaux recueillis ont fait l'objet d'une analyse (intra-cas et intercas), à l'aide de tableaux synthèses et de matrices élaborés suivant les recommandations de Huberman et Miles (1990). Selon ces derniers, ce type d'analyse de matériaux qualitatifs est particulièrement approprié pour les études de nature exploratoire.

\section{Présentation des résultats}

Le volume important de données recueillies rendrait difficile de présenter ici l'ensemble des analyses intra-cas effectuées pour chacune des entreprises. Dans une optique de parcimonie et de concision, nous privilégierons donc une approche

Revue internationale P.M.E., vol. 15, ns 3-4, 2002 


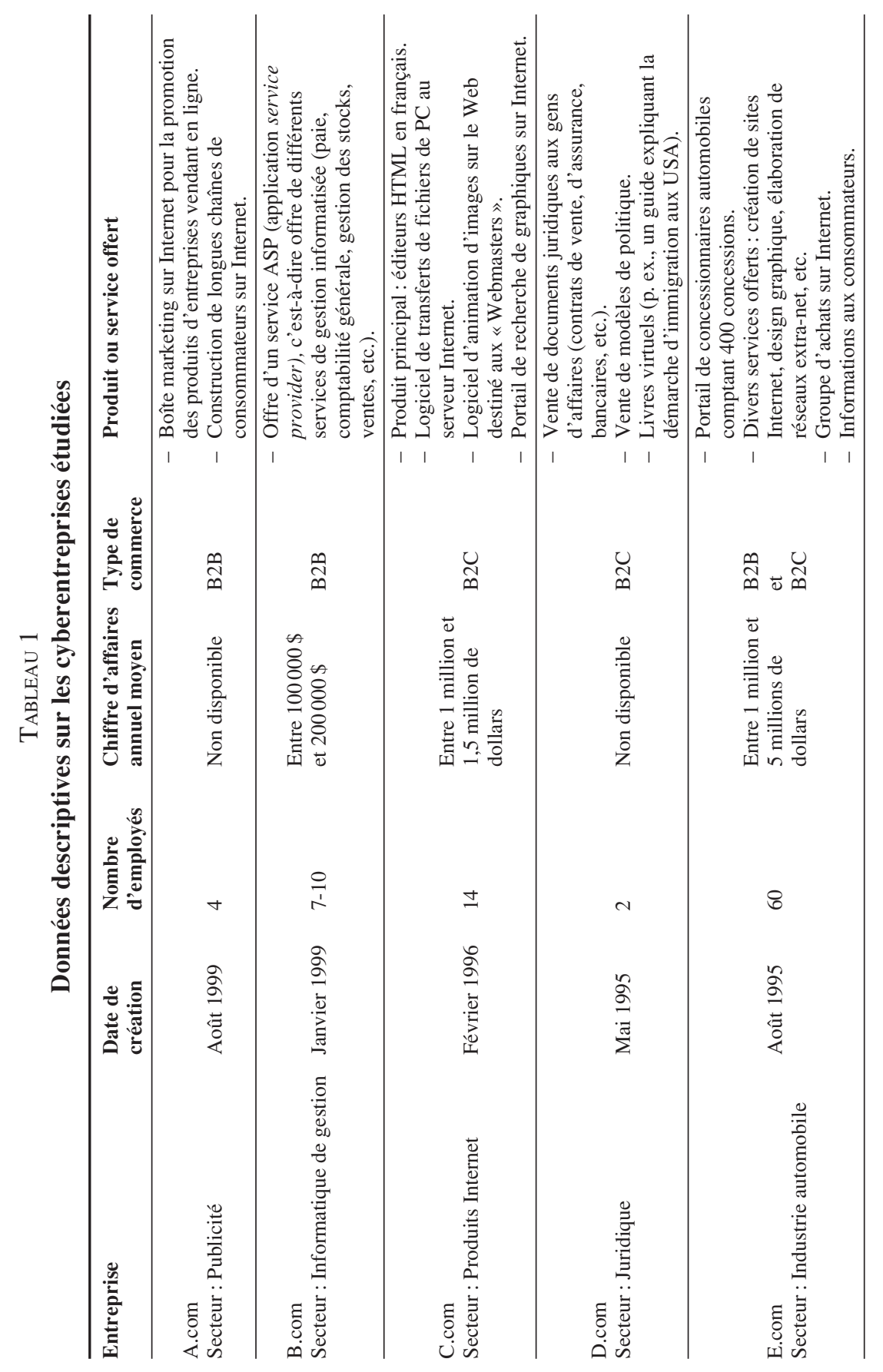

Revue internationale P.M.E., vol. 15, nºs 3-4, 2002 
comparative ou intégrative pour l'analyse des données. Le tableau 1 permet de visualiser le portrait d'affaires de chacune des entreprises étudiées. Pour assurer la confidentialité des sujets ayant accepté de participer à la recherche, nous avons utilisé des noms fictifs pour chaque entreprise. Signalons, en outre, que le chiffre d'affaires annuel moyen n'a pu être obtenu de toutes les entreprises.

\subsection{Profil sociodémographique}

Le tableau 2 présente le profil des cinq entrepreneurs propriétaires des cyberentreprises étudiées. Comme on peut le constater, tous ces entrepreneurs ont un cursus de formation avancé puisqu'ils sont tous détenteurs d'au moins un diplôme de $1^{\text {er }}$ cycle universitaire et que deux d'entre eux sont même titulaires d'une maitrise. Un tel constat vient appuyer les propos de Julien (2000) qui affirme qu'un des changements les plus frappants chez l'entrepreneur depuis les deux dernières décennies se révèle dans la qualité de sa formation. Selon ce dernier, un sondage réalisé en 1997 auprès de 547 dirigeants de PME révèle en effet que près de la moitié d'entre eux étaient détenteurs d'un diplôme universitaire et près de $20 \%$ étaient titulaires d'une maîtrise ou d'un doctorat. S'intéressant aux cyberentrepreneurs, il est plausible de croire que la formation puisse avoir tendance à être encore plus élevée dans ce secteur particulier. En effet, la totalité des répondants ont une formation universitaire et près de la moitié sont détenteurs d'un diplôme de $2^{\mathrm{e}}$ cycle.

On constate également que le succès des entreprises étudiées est loin d'être le fruit de savantes improvisations ou de «coups de génie», comme semblent souvent le laisser croire les «histoires d'une réussite » publiés dans les journaux et les revues d'affaires. En effet, tous les cyberentrepreneurs ont une solide expérience dans le domaine de spécialisation de leur entreprise. Il est intéressant de noter que l'entrepreneur propriétaire de A.com est le seul à n'avoir aucune formation en technologies de l'information. Sa force est d'abord et avant tout le marketing et l'innovation réside beaucoup plus dans le fait d'apporter un concept particulièrement original de publicité sur Internet, plutôt que d'utiliser les TI de façon créative. Cet entrepreneur s'est d'ailleurs adjoint des employés plus habiles que lui dans le domaine de l'informatique. Contrairement aux quatre autres cas, on ne peut le qualifier d'entrepreneur technologique.

On remarque aussi que certains entrepreneurs ont concilié deux formations complémentaires. Ainsi, le propriétaire de B.com, qui est à la fois détenteur d'une formation en droit et d'études collégiales en informatique, a conjugué ses deux types d'expertise, ce qui lui permet de créer et de distribuer des produits à caractère juridique en exploitant les technologies de l'information et de la communication. De façon plus générale, cette solide expérience des entrepreneurs vient corroborer l'un des facteurs de succès importants de création d'entreprise énoncés dans un

Revue internationale P.M.E., vol. 15, ns 3-4, 2002 


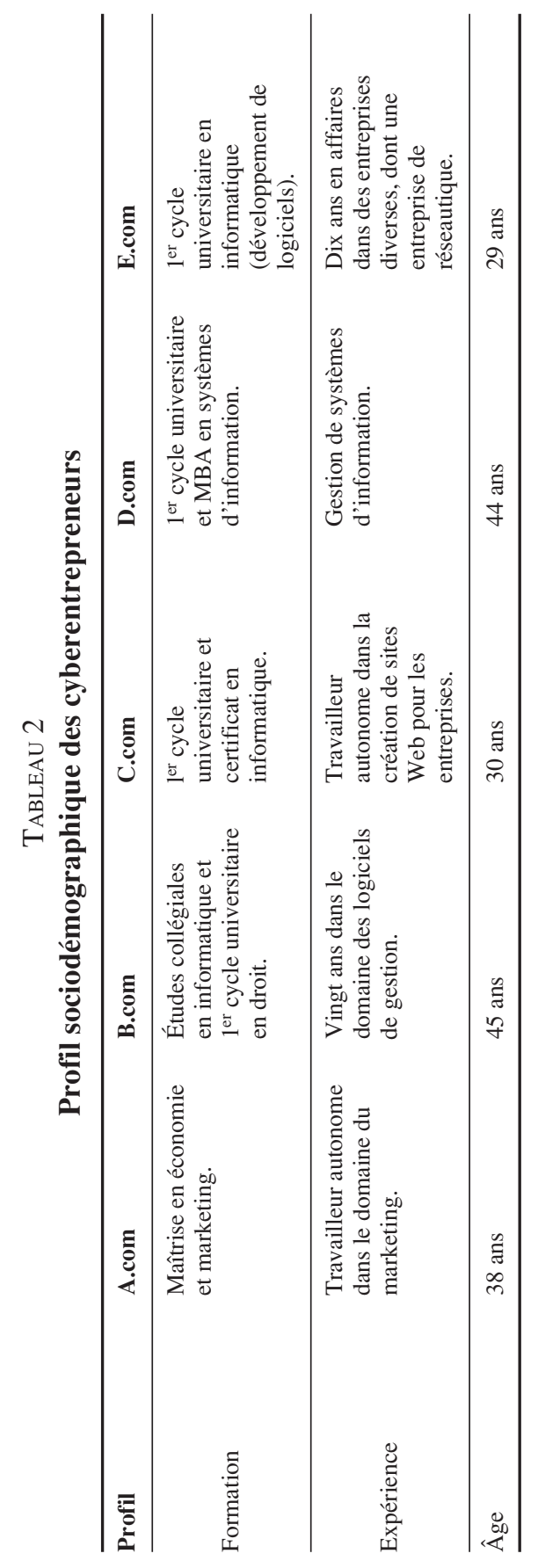

Revue internationale P.M.E., vol. 15, nos 3-4, 2002 
contexte plus traditionnel, soit la nécessité de posséder une expertise dans le domaine concerné par le projet d'affaires (Gasse et Carrier, 1992; d'Amboise, 1989; Filion, 2001). Enfin, on constate qu'aucun des cyberentrepreneurs n'est très jeune, ce qui vient encore ici détruire le mythe du jeune génie frais émoulu de l'université qui fait fortune en lançant une entreprise sur Internet.

\subsection{Processus entrepreneurial}

Il apparait également intéressant de pouvoir mettre au jour le processus emprunté par les cyberentrepreneurs pour créer leur entreprise. À cet effet, dans une première partie de l'entrevue, les sujets ont été amenés à raconter en détail l'histoire de la mise sur pied de leur entreprise, à partir du moment où les premières idées leur sont venues jusqu'à la création effective de l'entreprise. Une analyse de ces récits a permis de reconstruire la démarche empruntée. Après comparaison des cinq processus reconstitués, les cinq cyberentrepreneurs apparaissent être passés par les mêmes phases pour mettre sur pied leur entreprise. Ces phases sont les suivantes :

1) Émergence de l'idée d'affaires;

2) Analyse des besoins du marché;

3) Identification de l'occasion d'affaires;

4) Étude de faisabilité;

5) Recherche de soutien;

6) Création de l'entreprise.

L'annexe I présente la fiche synthèse élaborée pour l'analyse des résultats et fait état des démarches, actions ou attitudes reliées à chacune de ces étapes, tandis que la figure 1 permet pour sa part de visualiser le processus de création des cinq entreprises étudiées. Examinons de plus près la description de chacune de ces étapes.

\subsection{1. Émergence de l'idée d'affaires}

Cette première phase du processus entrepreneurial est marquée par une amorce de vision initiale, d'une idée paraissant porteuse au départ. L'entrepreneur de A.com, réalisant les possibilités de faire de la publicité dans la nouvelle économie et conscient des capacités offertes par les nouvelles TI, jongle avec l'idée de créer une formule de promotion basée sur le principe du bouche à oreille entre les consommateurs. L'entrepreneur de B.com est, pour sa part, littéralement «frappé » par une sorte de signal d'alerte à la suite d'une lecture sur la technologie Internet. Il en discute avec des collègues, amis et associés éventuels, et sont alors menés des

Revue internationale P.M.E., vol. 15, ns 3-4, 2002 
Figure 1

\section{Processus de création des entreprises étudiées}

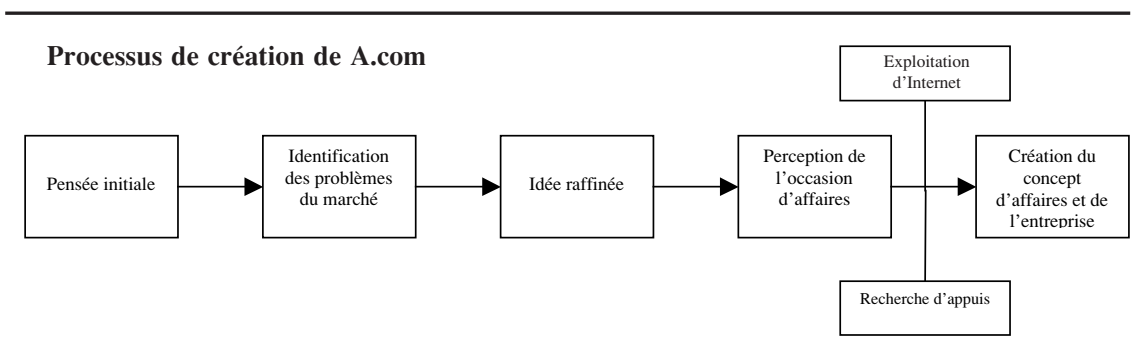

Processus de création de B.com et E.com

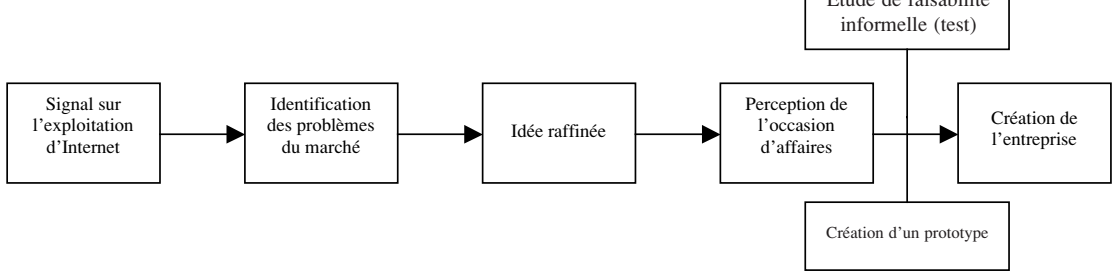

Processus de création de B.com et E.com

Découverte et exploitation d'Internet

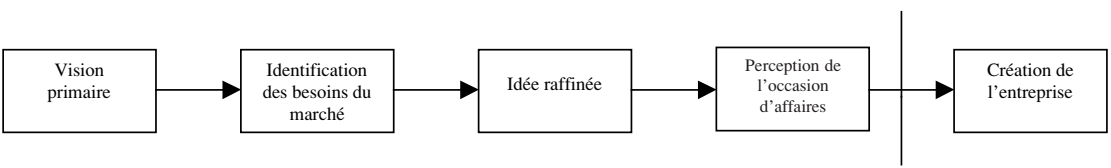

remue-méninges pour explorer en profondeur les différents produits ou services de son domaine pouvant être offerts par cette technologie. Quant à l'entrepreneur de C.com, son idée de départ l'a amené bien au-delà de ce qu'il a finalement sélectionné comme idée. Initialement,il désirait créer une galerie d'art sur un BBS (babillard électronique), puis sur un site Internet. Après maintes réflexions et discussions avec des experts, l'idée a évolué vers la création d'un éditeur HTML (hyper text markup language) en français pour les artistes et les graphistes.

Revue internationale P.M.E., vol. 15, $\mathrm{n}^{\text {os }} 3-4,2002$ 
L'entrepreneur de D.com a été frappé par un signal du marché, soit par la nécessité de développer un guide juridique et administratif pour les entrepreneurs potentiels, puis l'idée a évolué vers la possibilité de développer et de commercialiser plusieurs documents juridiques vendus séparément pour mieux répondre aux exigences des clients. Enfin, l'entrepreneur de E.com avait toujours œuvré dans le domaine de l'automobile compte tenu des activités professionnelles de son père. Passionné par les nouvelles technologies, et en particulier Internet, il s'est demandé s'il ne serait pas possible de révolutionner ce domaine avec un tel outil. L'idée de départ, créer un centre d'achats sur Internet, a lentement évolué vers un public plus important, celui des concessionnaires automobile. L'idée d'un portail est omniprésente, mais il tergiverse longtemps et consulte plusieurs personnes pour préciser la nature de l'offre.

\subsubsection{Analyse des besoins}

Dans les cinq cas, les entrepreneurs ont investi d'énormes efforts et multiplié les démarches pour déterminer les besoins des différentes clientèles pour les produits visés par leur idée initiale. En fait, se montrant tout aussi pragmatiques que les entrepreneurs technologiques décrits par Blais et Toulouse (1992), tous ont abordé cette étude des besoins en tentant d'identifier tous les problèmes vécus par les consommateurs ou entreprises clientes visés. Bien sûr, les aspects techniques reliés aux démarches empruntées pour explorer et connaître les problèmes et leur importance relative pour les entreprises ou les consommateurs concernés ont varié selon le secteur. Dans certains cas, ce sont des individus qui ont été interrogés alors que, dans d'autres, ce sont des gens d'affaires, des associations diverses (consommateurs, détaillants, concessionnaires, firmes-conseils, etc.). Il est intéressant de constater que les entrepreneurs ont accordé autant d'importance à l'analyse des besoins. En fait, cette phase diffère ici sensiblement de la phase d'étude de marché rencontrée dans des processus entrepreneuriaux plus traditionnels. Dans une analyse de marché typique, le produit ou le service est déjà bien connu ; il s'agit de vérifier l'existence d'un marché suffisant et les conditions qui inciteraient des clients éventuels à se le procurer. Nos entrepreneurs ont plutôt utilisé cette étape pour raffiner leur idée d'affaires initiale.

\subsubsection{Identification de l'occasion d'affaires}

L'exploitation d'une occasion d'affaires constitue une phase fondamentale du processus entrepreneurial. Comme on l'a vu précédemment, certains en font même le fondement de l'entrepreneuriat ; c'est le cas de Kirzner (1979) ainsi que de Shane et Venkataraman (2000). Dans la même veine, Stevenson, Roberts et Grousbeck (1985) affirment que l'entrepreneuriat repose d'abord et avant tout sur la perception d'une occasion d'affaires. Dans la plupart des cas étudiés, l'idée d'affaires se

Revue internationale P.M.E., vol. 15, ns 3-4, 2002 
concrétise principalement parce que les entrepreneurs ont investi beaucoup de temps pour découvrir les véritables besoins des marchés visés et ont conséquemment été les premiers à proposer une solution concrète qui est de surcroît innovatrice. Un autre facteur ayant grandement contribué à reconnaître l'occasion d'affaires est la connaissance de toutes les possibilités offertes par les nouvelles technologies, en particulier par l'Internet. Somme toute, les solutions proposées par ces entrepreneurs étaient innovatrices en elles-mêmes, mais c'est l'outil Internet qui les a rendues réalisables et profitables.

\subsection{4. Étude de faisabilité}

L'occasion d'affaires étant établie, les entrepreneurs ont tous pris la décision de la tester et d'examiner la faisabilité de leur projet. Cependant, cette analyse de la faisabilité a pris des formes différentes suivant les choix effectués par chacun des cinq porteurs de projet. L'entrepreneur de A.com a carrément procédé à l'élaboration d'un plan d'affaires, suivant les étapes classiques recommandées pour une telle démarche. Convaincu a priori de la pertinence de son projet, il a jugé qu'il s'agirait pour lui d'un instrument stratégique crucial lors d'une recherche de financement ultérieur.

L'entrepreneur de B.com, après plusieurs séances de remue-méninges avec différents groupes et beaucoup de recherche et développement, a élaboré un prototype de son produit qu'il a ensuite testé auprès d'un certain nombre d'entreprises clientes potentielles. Cette démarche a abouti à la conclusion de quelques contrats avec des PME ayant participé à l'expérimentation, ce qui est venu confirmer la faisabilité du projet. Par conséquent, l'entreprise avait déjà obtenu des contrats de service avant même d'être officiellement créée. L'entrepreneur de l'entreprise C.com a d'abord procédé à une vaste et importante étude de marché auprès des artistes et, dans un deuxième temps, a dressé un plan d'affaires (pour sa recherche éventuelle de financement). L'entrepreneur de D.com n'a pas fait d'étude de marché ni d'étude de faisabilité ; il a conçu le produit et lancé la plate-forme afin de la tester auprès de vrais clients, tout en se dotant d'un plan d'action pour corriger le tir rapidement et continuellement tout au long de l'opération. Enfin, l'entrepreneur de E.com a testé le potentiel d'Internet dans l'industrie automobile à travers une importante étude de consultation qu'il a menée auprès d'un nombre considérable de concessionnaires.

Après cette étape décisive qui venait confirmer la faisabilité d'exploitation de l'occasion d'affaires répérée, les cinq entrepreneurs étaient fermement décidés à créer leur entreprise. Cependant, il leur fallait trouver du financement et obtenir différents types de soutien.

Revue internationale P.M.E., vol. 15, nos 3-4, 2002

(C) 2003 - Presses de l'Université du Québec

Édifice Le Delta I, 2875, boul. Laurier, bureau 450, Sainte-Foy, Québec G1V 2M2 • Tél. : (418) 657-4399 - www.puq.uquebec.ca

Tiré de : Revue internationale P.M.E., vol. 15, nos 3-4, sous la direction de Pierre-André Julien. 


\subsubsection{Recherche de soutien}

Tout comme pour l'étape précédente du processus cyberentrepreneurial, cette étape a pris différentes formes suivant la nature des projets, les besoins inhérents à leurs caractéristiques et les ressources des entrepreneurs concernés. L'entreprise A.com a dû se mettre à la recherche de partenaires pour le financement de l'entreprise ainsi que d'associés pour préciser le concept d'affaires sous ses aspects technologiques. On se rappellera que le créateur de cette entreprise est le seul à avoir des compétences dans un domaine autre que les nouvelles technologies, soit en marketing. L'entrepreneur de B.com a cherché une société qui accepterait d'intégrer la nouvelle entreprise et de participer financièrement à son incubation. L'entrepreneur de C.com a d'abord cherché du financement directement auprès des banques plutôt que de tenter de trouver des associés-investisseurs ; il a également conclu des partenariats avec des portails de commerce électronique sur le Web pour assurer la promotion de son entreprise. L'entrepreneur de D.com, comme il n'avait pas de problème de financement, a concentré ses efforts dans la recherche de partenaires pour le support technologique de l'affaire. Enfin, l'entrepreneur de E.com a établi des partenariats avec des associations (automobiles et concessionnaires) et certaines d'entre elles ont accepté de participer au financement de son entreprise ; une partie du financement de son projet a également été assurée par des anges.

Pour conclure cette section, il importe de préciser que si les phases de ce processus ont été les mêmes pour tous les projets étudiés, leur chronologie a différé (comme le démontre la figure 1); soulignons que la durée consacrée à ces étapes a aussi varié. Toutefois, ce qui frappe plus particulièrement, c'est le fait que ce sont les étapes précédant l'étude de faisabilité qui ont été jugées comme les plus importantes, les plus énergivores mais aussi les plus critiques dans l'ensemble du processus.

Tenant compte à la fois des différences dans la chronologie des étapes et des variations dans la façon d'aborder chacune de ces dernières, nous proposons un modèle de création de la cyberentreprise qui intègre mieux ces écarts et qui raffine davantage le processus (voir figure 2).

\subsection{Les bases de la compétitivité de la cyberentreprise}

Contrairement aux résultats de la section précédente qui présente un processus de création reconstruit à partir du récit des sujets, ce sont les cyberentrepreneurs euxmêmes qui, avec des questions plus précises, ont été amenés à identifier les facteurs de compétitivité de leur entreprise. En d'autres termes, nous désirions savoir comment, dans un contexte de forte concurrence, ces derniers parvenaient à tirer leur épingle du jeu. Les sous-sections suivantes présentent donc les principaux éléments qui ont été relevés par les sujets comme étant à la base même de leur compétitivité.

Revue internationale P.M.E., vol. 15, ns 3-4, 2002 


\subsubsection{Exploitation des possibilités de l'entreprise-réseau}

La cyberentreprise parait de prime abord pouvoir fonctionner à partir d'une structure simple, au sens de Mintzberg (1981). Cependant, ses modes d'opérations peuvent lui permettre de prendre la forme d'une entreprise réseau dans le cyberespace, et ce, grâce à son infrastructure Internet et en extranets (Raymond et Blili, 2000). C'est le cas des entreprises A.com, B.com et E.com (dont les activités reposent sur le commerce électronique de type B2B), qui lient les clients par l'Internet en formant des communautés virtuelles connectées en réseau autour d'un produit, d'un service ou d'un objectif commun. Or, plus le nombre de visiteurs sur le site Web d'une communauté augmente, plus le réseau produit de la valeur ajoutée pour ses membres.

\subsubsection{Motivation du personnel par la participation au capital de l'entreprise}

Afin de retenir leur personnel et le motiver à être à la fois créatif et productif, la plupart des entrepreneurs interrogés offrent à leurs employés la possibilité de participer au capital de l'entreprise. Cette participation prend cependant des formes différentes. Dans certains cas, cette offre est plus sélective puisque ce sont d'abord et avant tout les employés les plus compétents, en l'occurrence ceux qui sont principalement détenteurs du savoir stratégique sur l'exploitation des nouvelles technologies de l'information, qui se voient offrir des options de participation au capital-action de l'entreprise. D'autres cyberentrepreneurs choisissent plutôt d'encourager tous leurs employés à devenir actionnaires de l'entreprise, ce choix étant justifié par leur conviction qu'une telle approche les fidélise et augmente leur niveau d'engagement. L'une des entreprises utilise un moyen quelque peu différent de l'actionnariat, misant plutôt sur un mécanisme de partage collectif des bénéfices avec les employés, le but visé étant toujours ici de responsabiliser ces derniers et de les motiver à contribuer davantage aux efforts du groupe.

\subsubsection{Veille technologique et concurrentielle systématique}

Les entrepreneurs de la nouvelle vague ont des comportements stratégiques qui diffèrent sensiblement de l'entrepreneur traditionnel qu'on a souvent décrit comme plutôt réactif et ayant tendance à opter pour une planification stratégique moins formalisée (Blili et Raymond, 1993). Les cyberentrepreneurs interrogés disent accorder une grande importance à la planification stratégique, planification qui se traduit par des plans d'actions structurés et continuellement révisés en fonction de la dynamique et de la turbulence de leurs marchés. Ils se comportent en décideurs proactifs qui tirent leur inspiration d'une bonne connaissance de tout ce qui se passe dans leur domaine grâce à une veille technologique et concurrentielle

Revue internationale P.M.E., vol. 15, $\mathrm{n}^{\text {os }} 3-4,2002$ 
systématique. L'un des entrepreneurs affirmait d'ailleurs à ce sujet: «Il ne faut jamais sous-estimer la compétition ni se sur-estimer. Nous évoluons dans un milieu où les technologies changent presque quotidiennement. Nous dépendons beaucoup des percées technologiques et il nous faut continuellement améliorer notre offre et trouver des façons de faire plus efficaces. » Un autre mettait l'accent sur la surveillance vigilante de ce que font tous les concurrents actuels ou potentiels de façon à ne jamais se laisser égaler ou surpasser par ces derniers. Par la nature même de leurs activités, les cyberentrepreneurs se sentent d'ailleurs mieux équipés que d'autres pour exercer une veille stratégique efficace, maîtrisant très bien les outils de veille requis.

\subsubsection{Utilisation maximale des technologies de l'information}

Les recherches sur l'utilisation des technologies de l'information par les PME ont démontré que plusieurs n'utilisent pas ces technologies pour intégrer la chaîne de valeur de l'entreprise (production, marketing et ventes, logistique amont et aval, etc.). En outre, les systèmes qu'elles ont tendance à utiliser le sont pour des fins plutôt administratives que stratégiques (Raymond et Blili, 1998). Il en va tout autrement dans les cyberentreprises étudiées qui évidemment ne négligent pas le rôle stratégique et concurrentiel des TI. Dans la majorité de ces entreprises, les TI sont intégrées dans l'organisation de l'ensemble des fonctions de l'entreprise, qu'il s'agisse de la production, du marketing, de la vente, de la gestion financière et même de la totalité des transactions qu'ils effectuent avec leurs systèmes clients respectifs.

\subsubsection{Bon positionnement sur le marché grâce à des stratégies ciblées}

Dans tous les cas étudiés, les entreprises sont fortement positionnées sur leur marché grâce à des stratégies porteuses de succès. Plus concrètement, quatre des cinq entreprises occupent une position de leader sur leur marché actuel et la cinquième a réussi à se tailler une place dans un créneau qui intéresse peu les éditeurs de logiciel œuvrant dans le domaine.

L'entreprise A.com a opté pour une stratégie de domination par les coûts à travers la réalisation d'économies d'échelle dues à une forte croissance. L'utilisation d'Internet est la principale source d'avantage concurrentiel étant donné sa facilité d'accès, son coût négligeable et sa force en tant que réseau mondial. Les entreprises B.com, D.com et E.com ont plutôt misé sur une stratégie de différenciation basée soit sur la qualité du service, soit sur l'image et la qualité des produits. Quant à C.com, elle réussit grâce à une stratégie de niche. L'entrepreneur, lors de son analyse des besoins, a découvert que le marché des éditeurs HTML était principalement

Revue internationale P.M.E., vol. 15, ns 3-4, 2002 


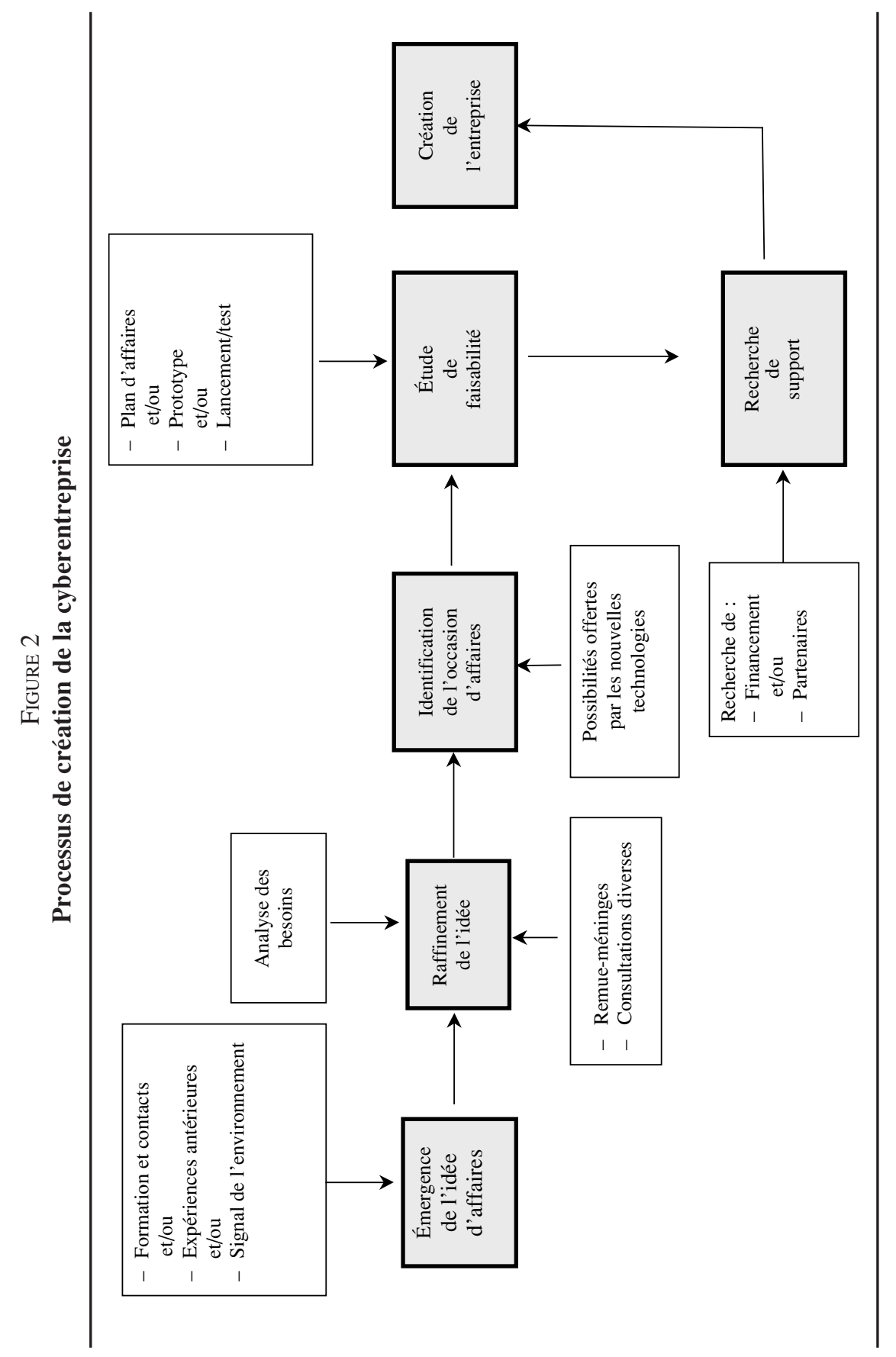

Revue internationale P.M.E., vol. 15, nos 3-4, 2002 
détenu par des leaders déjà bien établis, en l'occurrence des multinationales telles que Microsoft qui possèdent depuis des années le quasi-monopole du marché mondial pour ce type de produits informatiques. L'entreprise a donc concentré ses efforts à la production de logiciels en français, un segment du marché peu susceptible d'intéresser les leaders déjà présents dans le domaine. En plus d'être convaincus de la pertinence et de la qualité des stratégies empruntées, tous les entrepreneurs interrogés se sont dits persuadés qu'ils devaient leur position avantageuse actuelle à leur capacité d'innover et d'être les premiers à offrir leur produit ou leur service.

\subsubsection{Une préoccupation importante: les mesures de sécurité}

Comme le soulignent Bret et Champeaux (2000), la sécurité des réseaux informatiques est devenue un enjeu stratégique. Le cyberespace est en effet un univers soumis à certains risques liés à la divulgation de l'information «riche» à travers l'Internet et les extranets. En outre, sont présents des risques liés au piratage des produits et à l'attaque des sites d'entreprise. Les entreprises peuvent voir leurs produits ou services copiés sans autorisation ou subir des tentatives de fraudes. Tous les cyberentrepreneurs interrogés se sont montrés très conscients des menaces liées à ces risques, menaces pouvant aller jusqu'à mettre en péril leur propre compétitivité et sécurité ainsi que celles de leurs partenaires ou de leurs clients. Ils ont donc tous prévu des mesures de sécurité effectives, mesures qui diffèrent suivant les besoins. Le tableau 3 présente les mesures de sécurité privilégiées et mises en œuvre par les cinq entreprises concernées.

TABLEAU 3

Mesures de sécurité adoptées

\begin{tabular}{ccccc}
\hline A.com & B.com & C.com & D.com & E.com \\
\hline $\begin{array}{c}\text { Coupe-feu pour } \\
\text { protéger le site } \\
\text { et encryptage } \\
\text { des données } \\
\text { transférées à }\end{array}$ & $\begin{array}{c}\text { Coupe-feu pour } \\
\text { les réseaux } \\
\text { privés } \\
\text { et protégés } \\
\text { pour les clients. }\end{array}$ & $\begin{array}{c}\text { Coupe-feu } \\
\text { pour protéger } \\
\text { les produits, } \\
\text { système de } \\
\text { travers le Web. } \\
\text { et contrôle } \\
\text { rigoureux des } \\
\text { commandes } \\
\text { des clients. }\end{array}$ & $\begin{array}{c}\text { Coupe-feu pour } \\
\text { protéger les } \\
\text { produits et } \\
\text { système de } \\
\text { paiement } \\
\text { sécurisé. }\end{array}$ & $\begin{array}{c}\text { Coupe-feu } \\
\text { pour la protection } \\
\text { du portail et } \\
\text { encryptage } \\
\text { des données } \\
\text { transférées à } \\
\text { travers les } \\
\text { réseaux privés. }\end{array}$ \\
\hline
\end{tabular}




\section{Conclusion}

Les résultats de cette recherche apportent un éclairage intéressant sur une nouvelle forme entrepreneuriale susceptible de prendre de l'ampleur avec l'avènement de la nouvelle économie. Ses apports les plus importants résident certainement dans les constats effectués sur le processus de création de la cyberentreprise, par la proposition d'un modèle intégratif des pratiques observées à cet effet, et également dans une connaissance accrue des stratégies compétitives privilégiées par les cyberentrepreneurs ainsi que sur d'autres facteurs qu'ils associent à la compétitivité de leur entreprise.

Outre ces résultats importants, la recherche nous permet de mieux connaître l'entrepreneuriat de valorisation et l'entrepreneuriat d'aventure. Bruyat et Julien (2000) ont en effet avancé que l'entrepreneuriat pouvait prendre quatre formes différentes, selon l'importance intrinsèque de l'innovation ou de la valeur nouvelle créée par l'entrepreneur, et l'importance de cette création de valeur pour l'entrepreneur lui-même. L'entrepreneuriat de reproduction crée très peu de nouvelle valeur et entraîne peu de changement chez le créateur. L'entrepreneuriat d'imitation crée peu de valeur nouvelle, permet au créateur de changer, parfois de façon radicale, sa propre situation : l'essaimage en est un bon exemple. L'entrepreneuriat de valorisation est souvent le fait d'un entrepreneur ayant une idée nouvelle et créant une valeur nouvelle. L'entrepreneuriat d'aventure est celui qui crée véritablement une nouvelle valeur, un produit vraiment nouveau, suscitant souvent un changement d'importance dans l'économie. À ce titre, les entrepreneurs de A.com et de D.com ne proposent pas nécessairement un nouveau produit, mais on peut certainement parler d'un changement radical dans la façon de le proposer. On peut donc les considérer comme des entrepreneurs de valorisation. Quant aux entrepreneurs de B.com, C.com et E.com, ils offrent un nouveau produit sur le marché tout en innovant sur le plan technologique dans la façon de le distribuer: ce sont des entrepreneurs d'aventure. Comme le souligne Julien (2000), ces deux derniers types d'entrepreneurs sont ceux qui représentent le mieux l'essence de l'entrepreneuriat véritable; ils sont aussi les plus rares dans l'univers actuel des PME.

L'étude des cyberentreprises présentée ici nous a ainsi permis de produire des connaissances initiales sur cette importante génération de nouveaux entrepreneurs / innovateurs, prélude à des recherches subséquentes sur ce phénomène. Un autre apport non négligeable de cette recherche est qu'elle a mis au jour les conditions entourant l'émergence d'une idée d'affaires et surtout le processus d'exploration de l'occasion d'affaires qui en précède nécessairement l'identification. Vesper (1980) ainsi que Long et McMullen (1984) ont souligné toute l'importance de cette exploration d'une idée d'affaires. En outre, certains chercheurs, dont Cooper (1981) et Koller (1988), se sont particulièrement intéressés au processus d'évaluation et de perception d'une occasion d'affaires. Cependant, comme le

Revue internationale P.M.E., vol. 15, nos 3-4, 2002 
signalent Hills et Shrader (1998), le processus d'exploration qui entraîne la reconnaissance formelle d'une occasion d'affaires reste encore très peu étudié. Notre étude a permis de constater que les cinq cyberentrepreneurs étudiés ont accordé beaucoup d'importance à cette phase cruciale et ont décrit de façon intéressante le chemin parcouru à cet effet. De tels éléments pourraient certainement inspirer des chercheurs intéressés par l'exploration d'une idée d'affaires et guider éventuellement les acteurs responsables de différentes formations en entrepreneuriat. Comme le relève Carrier (2000), les formations en entrepreneuriat se concentrent trop souvent sur les aspects techniques de la création d'entreprise (souvent par le biais du plan d'affaires) et négligent de former les candidats à la recherche d'une idée porteuse.

Il va sans dire que les résultats présentés ici ne peuvent être généralisés, compte tenu du nombre réduit de cas étudiés; cela constitue une limite de notre recherche. Cependant, dans un contexte où cette étude voulait appréhender un phénomène nouveau à la fois dans l'environnement des affaires et dans la recherche en gestion, une recherche exploratoire, permettant de documenter en profondeur un certain nombre de cas, s'imposait. Les résultats de cette recherche ont permis d'élaborer un modèle de création de la cyberentreprise et des propositions de facteurs de compétitivité susceptibles de guider l'élaboration d'hypothèses pour des recherches futures menées auprès de plus larges échantillons. 


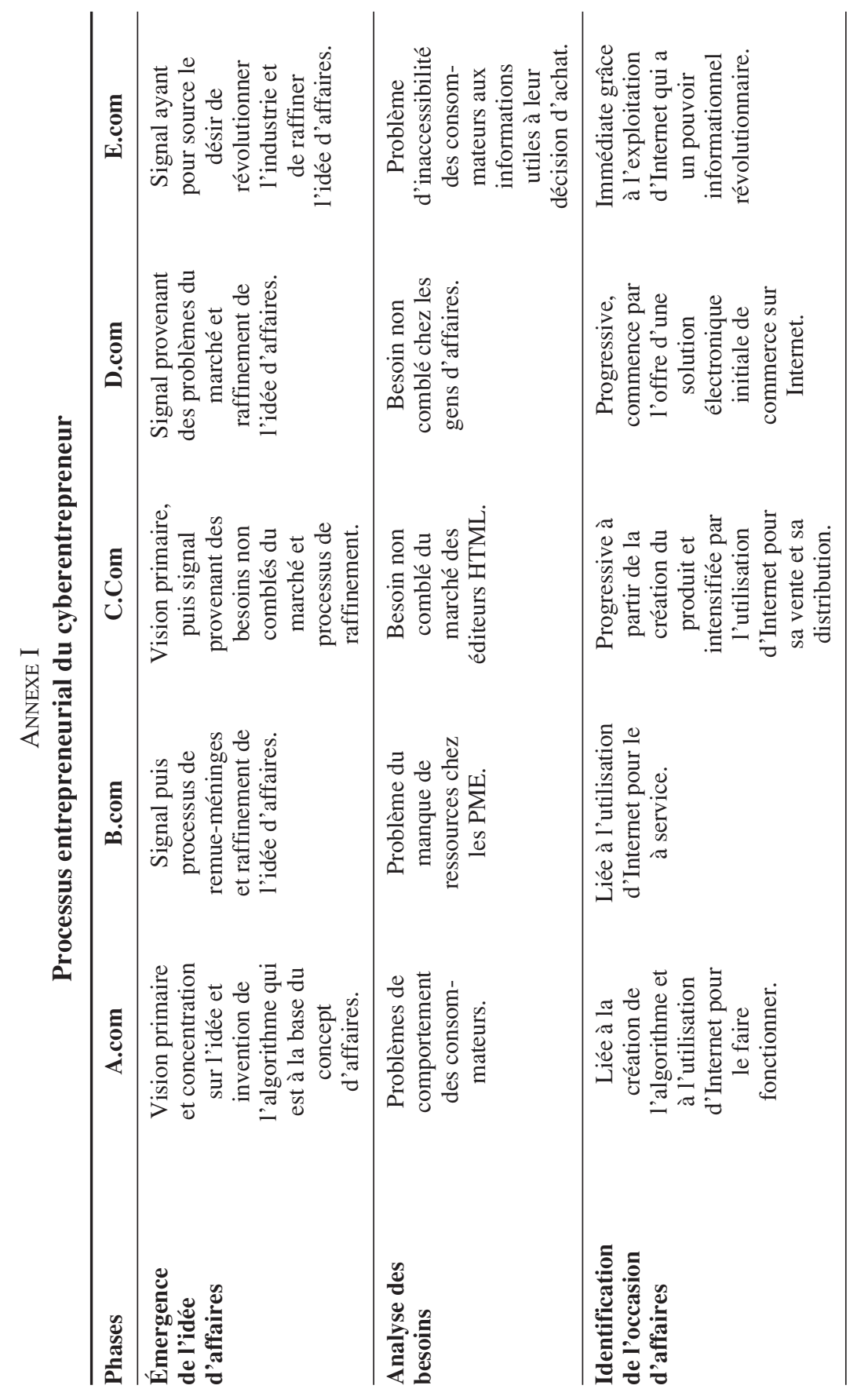

Revue internationale P.M.E., vol. 15, nºs 3-4, 2002 


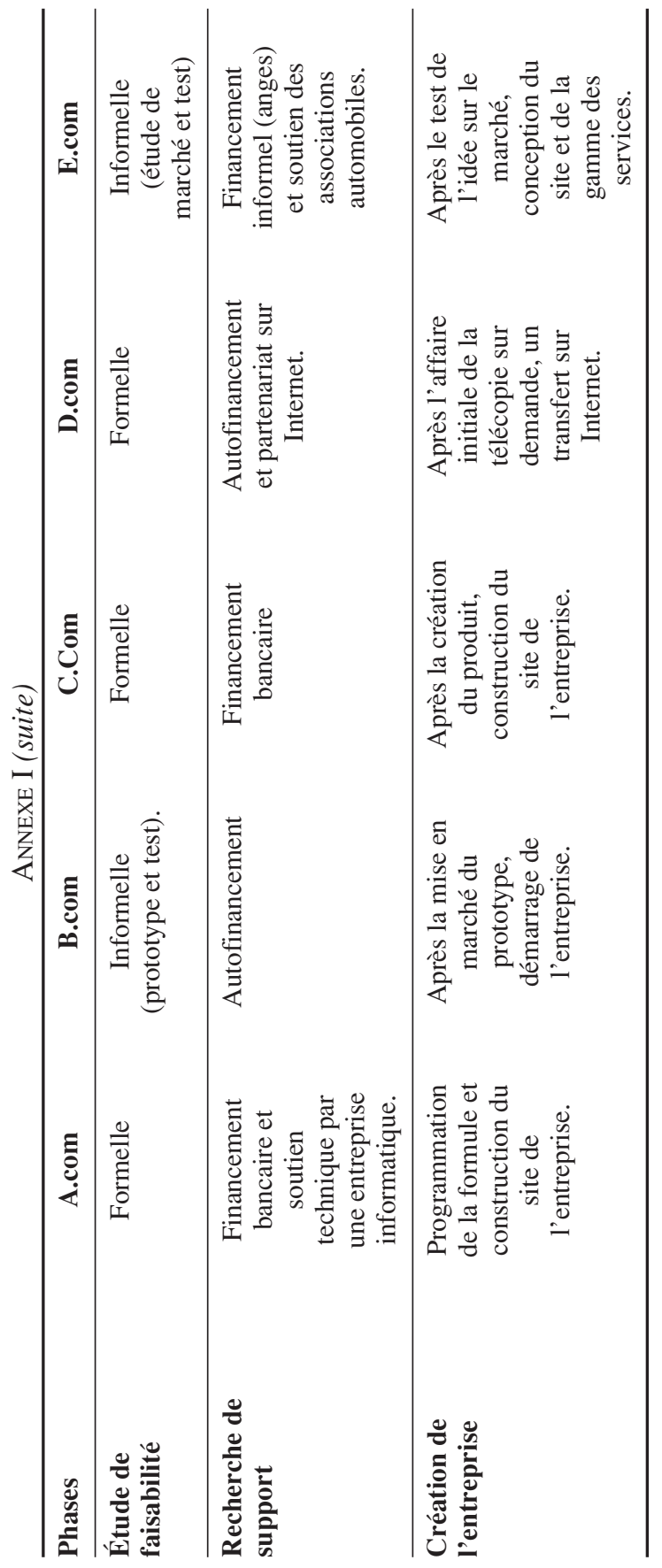

Revue internationale P.M.E., vol. 15, nos 3-4, 2002 


\section{Bibliographie}

BLAIR, R. et D.S. SCOTT (1979), The Technical Entrepreneur, Victoria, British Columbia, Press Porcepic Ltd.

BLAIS, R.A. et J.-M. Toulouse (1992), Entrepreneurship Technologique : 21 cas de PME à succès, Montréal, Fondation de l'Entrepreneurship / Publications Transcontinental.

BLILI, S. et L. RAYMOND (1993), «Information technology: threats and opportunities for SMEs », International Journal of Information Management, vol. 13, nº 6, p. 439-448.

BRET, C. et J. CHAMPEAUX (2000), La cyberentreprise : dix clés pour une approche intégrale des nouvelles technologies de l'information et de la communication dans l'entreprise, Paris, Dunod.

BRUYAT, C. et P.-A. JULIEN (2000), « Defining the field of research in entrepreneurship », Journal of Business Venturing, vol. 16, $\mathrm{n}^{\circ}$ 2, p. 17-27.

CARRIER, C. (2000), « Défis, enjeux et pistes d'action pour une formation entrepreneuriale renouvelée », Revue Gestion 2000, vol. 3, mai-juin, p. 149-163.

COOPER, A.C. (1981), «Strategic management: new ventures and small business », Long Range Planning, vol. 14. $\mathrm{n}^{\circ}$ 5, p. 39-45.

D’AmBoise, G. (1989), La PME canadienne : situation et défis, Québec, Presses de l'Université Laval, Institut de recherches politiques.

EISENHARDT, K.M. (1989), «Building theories from case study research », Academy of Management Review, vol. 14, $\mathrm{n}^{\circ}$ 4, p. 532-550.

FILION, L.J. (1996), «Two types of entrepreneurs: the operator and the visionary consequences for education», dans J.J. Pleitner (dir.), Renaissance of SMEs in a Globalized Economy, Saint-Gall, Verlag KMU/HSG.

FILION, L.J. (2000), « Typologie d'entrepreneurs : est-ce vraiment utile? » dans T. Verstraete (dir.), Histoire d'entreprendre: Les réalités de l'entrepreneuriat, Paris, Éditions EMS, p. 255-262.

FILION, L.J. (2001), Réaliser son projet d' entreprise, $3^{2}$ éd., Montréal, Les Éditions Transcontinental, coll. «Entreprendre ».

GASSE, Y. et C. CARRIER (1992), Gérer la croissance de sa PME, Montréal, Les Éditions de l'Entrepreneur.

HILLS, G.E. et R.C. SHRADER (1998), « Successfull entrepreneur's insights into opportunity recognition », Frontiers of Entrepreneurship Research, Wellesley, Babson College.

Holt, D.H. (1992), Entrepreneurship. New Venture Creation, Englewood Cliffs, NJ, Prentice-Hall.

Huberman, A.M. et M.B. Miles (1991), Analyse des données qualitatives, recueil de nouvelles méthodes, Bruxelles, De Boeck-Wesmael.

Julien, P.A. (2000), L'entrepreneuriat au Québec. Pour une révolution tranquille entrepreneuriale 1980-2005, Montréal, Les Éditions Transcontinental.

Julien, P.A. et M. MARChESNAY (1996), L'entrepreneuriat, Paris, Economica.

KIRZNER, I.M. (1979), Perception, Opportunity and Profit, Chicago, University of Chicago Press.

KOLLER, R.H. (1988), « On the source of entrepreneurial ideas », Frontiers of Entrepreneurship Research, Wellesley, Babson College.

Revue internationale P.M.E., vol. 15, nos 3-4, 2002 
LAFUENTE, A. et V. SAlas (1989), «Types of entrepreneurs and firms : the case of new Spanish firms », Strategic Management Journal, vol. 10, n 1, p. 17-30.

LONG, W. et W.E. MCMULlan (1984), « Mapping the new venture opportunity identification process », dans J.A. Hornaday (dir.), Frontiers of Entrepreneurship Research, Wellesley, Babson College, p. 567-590.

MAXWELl, J.A. (1996), Qualitative Research Design : An Interactive Approach, Beverly Hills, CA, Sage Publications.

MiLeS, R. et C.C. SNOw (1978), Organization Strategy, Structure and Process, New York, McGraw-Hill.

MinTZBERG, H. (1981), « Organiser l'entreprise : prêt-à-porter ou sur mesure ? », HarvardL'Expansion, été, p. 9-23.

O'Brien, J.A. (1999), Scientists as Entrepreneurs: Organizational Performance in Scientist-Started New Ventures, Boston, Kluwer Publisher.

RAYMOND, L. (2000), «Globalisation, économie du savoir et compétitivité : Un cadre de veille des tendances et enjeux stratégiques pour la PME», Gestion, vol. 25, n 2, p. 29-38.

RAYMOND, L. (2001), « Determinants of Web site implementation in small businesses », Internet Research, vol. 11, $\mathrm{n}^{\circ}$ 5, p. 411-422.

RAYMOND, L. et S. BLILI (1998), «Information systems », dans P.-A.Julien (dir.), The State of the Art in Small Business and Entrepreneurship, Aldershot, Angleterre, Ashgate, p. 300-329.

RAYMOND, L. et S. BLILI (2000), «Organizational learning as a foundation of electronic commerce in the network organization», International Journal of Electronic Commerce, vol. 5, no 2, p. 29-45.

SAMSON, K.J. (1990), Scientists as Entrepreneurs: Organizational Performance in Scientist-Started New Ventures, Boston, Kluwer Publishers.

SCOTT-Morton, M.S. (1995), «L'entreprise compétitive au futur, technologies de l'information et transformation de l'organisation », Ingénierie des systèmes d' information, Paris, Les Éditions d'Organisation.

Shane, S. et S. Venkataraman (2000), « The promise of entrepreneurship as a field of research », Academy of Management Review, vol. 25, n 1, p. 217-226.

SCHumPeter, J.A. (1959), The Theory of Economic Development, Cambridge, Harvard University Press.

SMITH, N.R. (1967), The Entrepreneur and His Firm: TheRelationship between Type of Man and Type of Company, East Lansing, Michigan State University Press, Graduate School of Business Administration.

SteVenson, H.M., J.J. RoberTs et H.I. GROUSBECK (1985), New Business Ventures and the Entrepreneur, Homewood, Irwin.

VERSTRAETE, T. (2000), Histoire d'entreprendre : Les réalités de l'entrepreneuriat, Paris, Éditions EMS.

Verstraete, T. (2001), «Entrepreneuriat: modélisation du phénomène », Revue de l'entrepreneuriat, vol. 1, $\mathrm{n}^{\mathrm{o}} 1$, p. 5-24.

Vesper, K.H. (1980), New Venture Strategies. Englewood Cliffs, NJ, Prentice-Hall.

Revue internationale P.M.E., vol. 15, ns 3-4, 2002 